\title{
Successful endoscopic closure of gastrocutaneous fistula using an over-the-scope clip
}

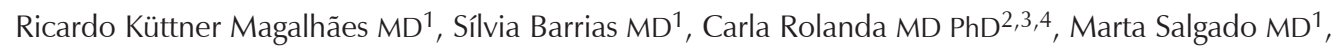 \\ Maria João Magalhães $M D^{1}$, Vitor Simões $M D^{5}$, Isabel Pedroto $M D^{1}$
}

\section{CASE PRESENTATION}

A 49-year-old man with heavy alcoholic habits was diagnosed with invasive squamous cell carcinoma of the amygdala (T4aN1Mx) for approximately one year. At that time, a percutaneous endoscopic gastrostomy (PEG) was performed, with placement of a 24 Fr tube using the Pull method, before chemotherapy and radiotherapy. The PEG tube was maintained for eight months, although the patient removed it accidentally several times, leading to successive replacements until it was not considered to be necessary and not reintroduced thereafter. During the following five months, the gastrocutaneous tract remained patent, with leakage of gastric contents, in spite of dietary precautions, omeprazole and domperidone. The external orifice of the fistula was apparent in the transition of the epigastrium to the left hypochondrium and the inner orifice on the anterior aspect of the medium/distal gastric body. A colostomy wafer was placed and bag adjusted to the external orifice, with consequent reduction of fistula output one week before endoscopic treatment was conducted. Argon plasma coagulation of the accessible part of the internal tract (Figure 1) followed by the application of an over-the-scope clip was performed (Figures 2 and 3). Post-procedure clinical success was achieved, with elimination of fistulous drainage. Complete closure of the internal opening of the fistula was confirmed at four weeks and three months, when an area of converging folds around the clip was observed (video [go to www.pulsus.com]). There was no recurrence over a 12 -month follow-up period.

\section{DISCUSSION}

Performing temporary PEG is indicated when transiently poor oral intake is anticipated (1). This patient did not present classical factors for gastrocutaneous fistula persistence after tube removal and its closure with conservative measures was not successful. In these cases, surgery is the traditional option. Endoscopic approaches to persistent gastrocutaneous fistula, such as fistula tract cauterization, fibrin glue, cyanoacrylate and conventional endoclips, have been described (2-4); however, some are associated with a significant failure rate. Placement of a colostomy wafer and bag into the external orifice is not usually performed, although in our experience it helps to decrease fistula output, despite not being considered essential. Cautery of the tract with silver nitrate from the external orifice and with electrocoagulation from the inner orifice is intended to ablate the epithelial lining of the tract (a factor that is considered to be crucial in the pathogenesis of persistent gastrocutaneous fistula) and to promote scar formation. In the present case, the use of silver nitrate was not warranted, and we chose argon plasma for coagulation of the tract. We did not believe it was necessary to use ancillary devices designed to approximate the defect edges before deploying the clip. Compared with conventional endoclips, over-thescope clips have the advantage of a higher rate of full-thickness closure (5), and appear to be a safe and effective alternative in gastrocutaneous fistula closure.

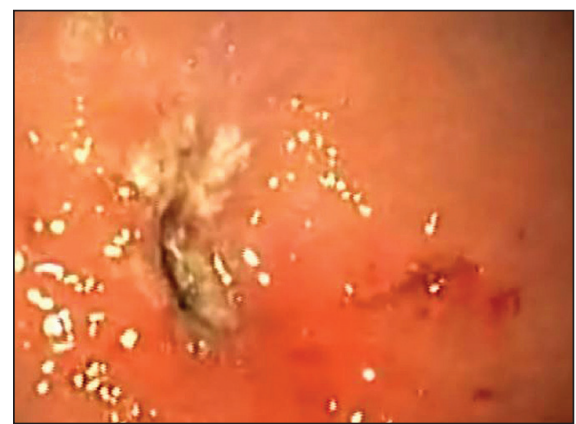

Figure 1) Endoscopic appearance of the internal orifice after argon plasma coagulation of the accessible tract

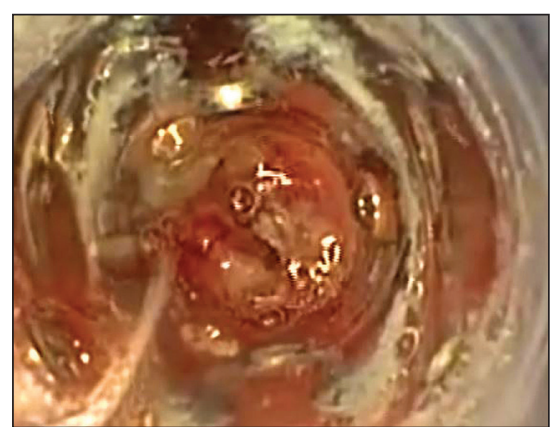

Figure 2) Application of the over-the-scope clip

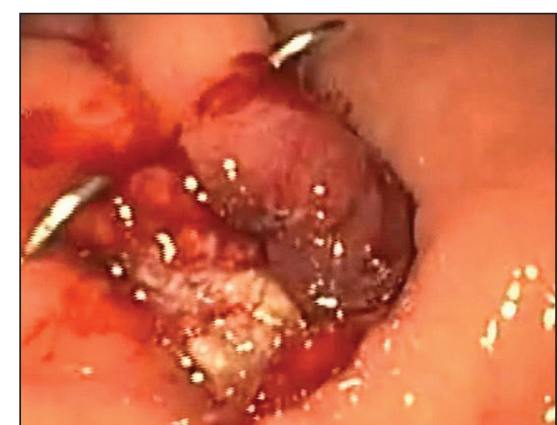

Figure 3) Endoscopic appearance immediately after clip deployment

\section{REFERENCES}

1. Westaby D, Young A, OToole P et al. The provision of a percutaneously placed enteral tube feeding service. Gut 2010;59:1592-605.

2. Akhras J, Tobi M, Zagnoon A. Endoscopic fibrin sealant injection with application of hemostatic clips: A novel method of closing a refractory gastrocutaneous fistula. Dig Dis Sci 2005;50:1872-4.

3. Duddempudi S, Ghevarina V, Singh M, et al. Treatment of persistently leaking post PEG tube gastrocutaneous fistula in elderly patients with combined electrochemical cautery and endoscopic clip placement. South Med J 2009;102:585-8.
4. Eskaros S, Ghevarina V, Krishnaiah M, et al. Percutaneous endoscopic suturing: An effective treatment for gastrocutaneous fistula. Gastrointest Endosc 2009;70:768-71.

5. von Renteln D, Vassiliou MC, Rothstein RI. Randomized controlled trial comparing endoscopic clips and over-the-scope clips for closure of natural orifice transluminal endoscopic surgery gastrotomies. Endoscopy 2009;41:1056-61.

${ }^{1}$ Department of Gastroenterology, Hospital Santo António, Centro Hospitalar Porto, Porto; ${ }^{2}$ Department of Gastroenterology, Hospital de Braga; ${ }^{3}$ Life and Health Sciences Research Institute (ICVS), School of Health Sciences, University of Minho, Braga; ${ }^{4}$ ICVS/3B's - PT Government Associate

Laboratory, Braga/Guimarães; ${ }^{5}$ Department of Surgery, Hospital Santo António, Centro Hospitalar Porto, Porto, Portugal

Correspondence: Dr Ricardo Küttner Magalhães, Rua Dr. Eduardo Santos Silva, 59, 5ํDto, 4200-282, Porto, Portugal. Telephone 96-411-9825,

e-mail rkuttner@gmail.com

Received for publication January 28, 2014. Accepted February 7, 2014 


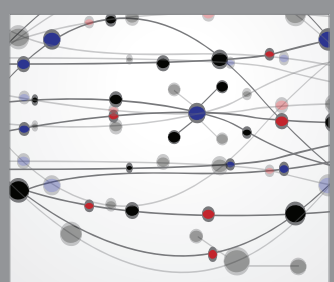

The Scientific World Journal
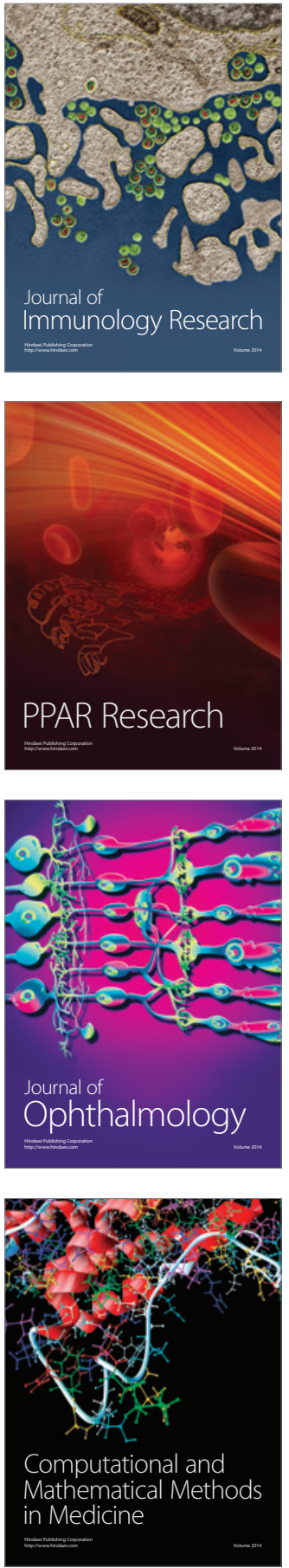

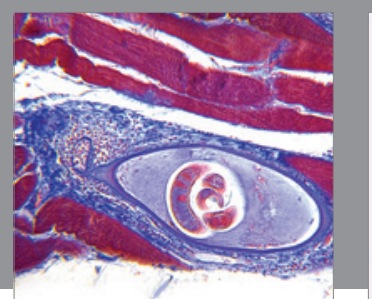

Gastroenterology Research and Practice

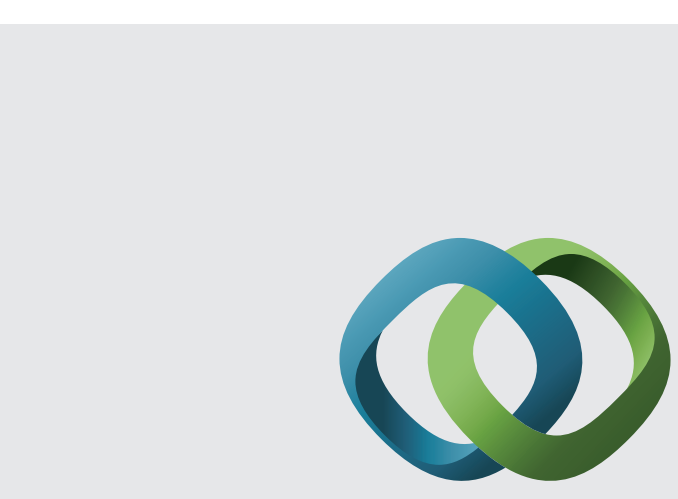

\section{Hindawi}

Submit your manuscripts at

http://www.hindawi.com
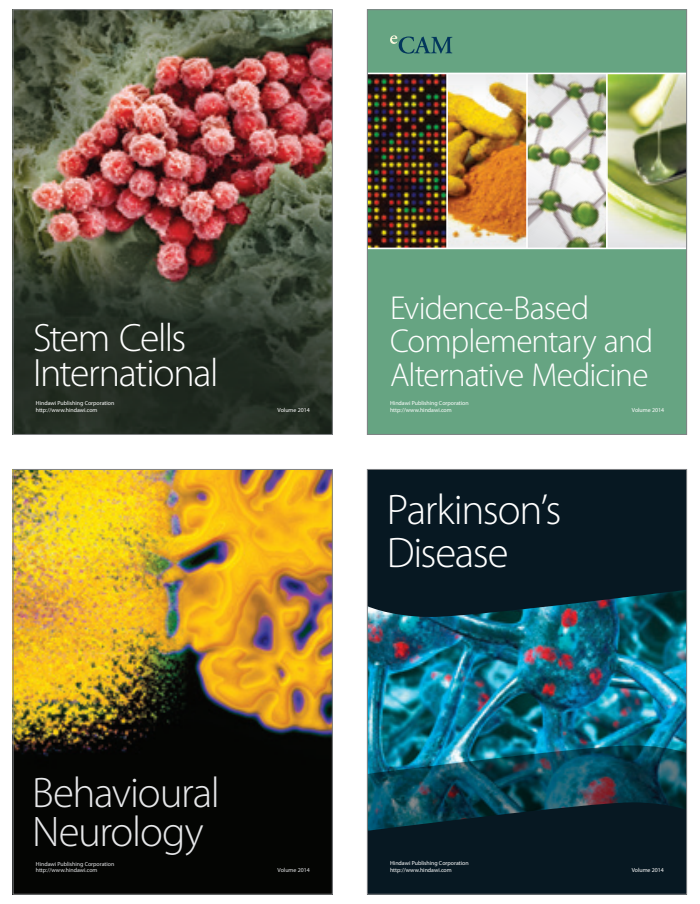
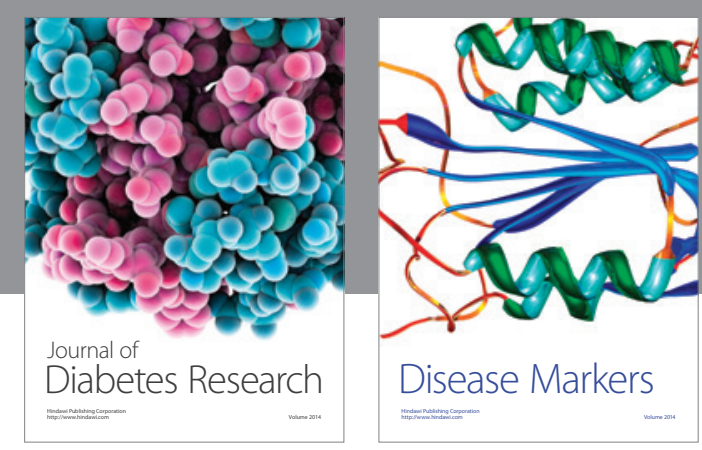

Disease Markers
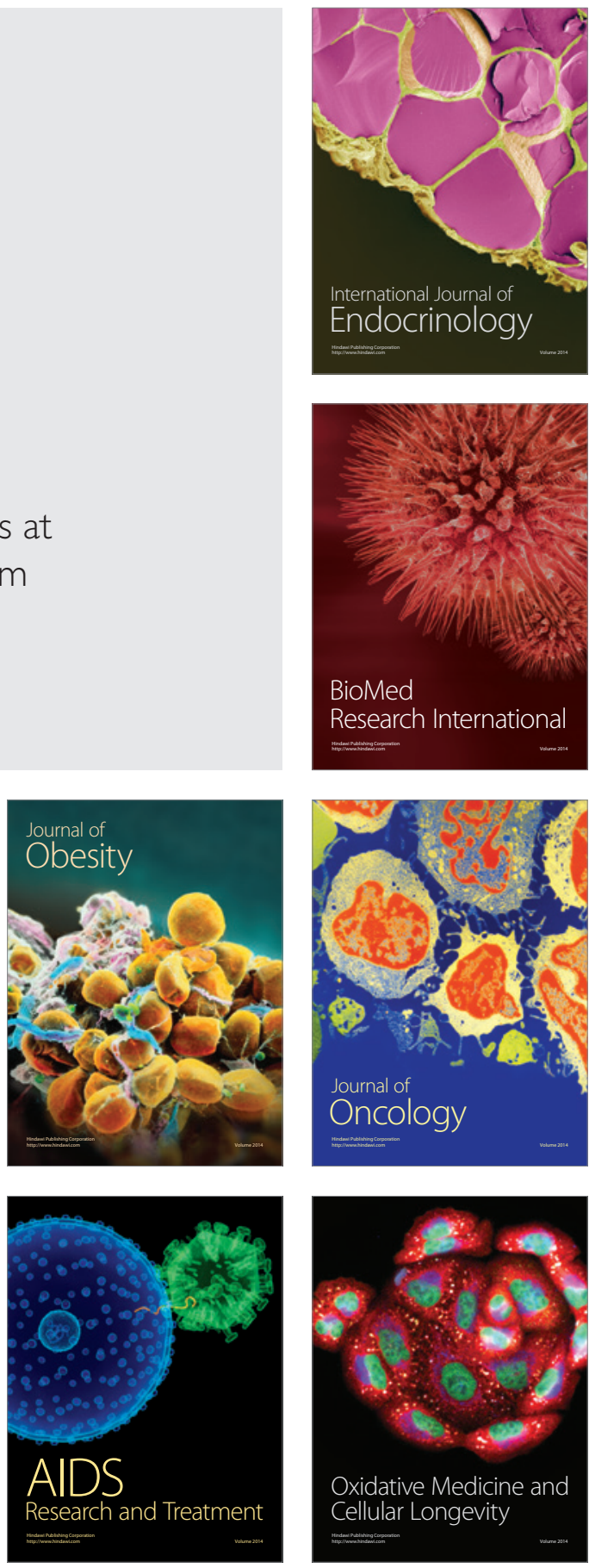